\title{
STM and DFT study on formation and characterization of Ba-incorporated phases on a Ge(001) surface
}

\author{
W. Koczorowski, ${ }^{1,{ }^{*}}$ A. Puchalska, ${ }^{2}$ T. Grzela, ${ }^{1}$ L. Jurczyszyn, ${ }^{2}$ S. R. Schofield,${ }^{3,4}$ R. Czajka, ${ }^{1}$ \\ N. J. Curson, ${ }^{3,5}$ and M. W. Radny ${ }^{1,6}$ \\ ${ }^{1}$ Institute of Physics, Poznan University of Technology, ul. Piotrowo 3, 60-965 Poznan, Poland \\ ${ }^{2}$ Institute of Experimental Physics, University of Wroclaw, pl. Maksa Borna 9, 50-204 Wroclaw, Poland \\ ${ }^{3}$ London Centre for Nanotechnology, UCL, 17-19 Gordon Street, London, United Kingdom \\ ${ }^{4}$ Department of Physics and Astronomy, UCL, London, WC1E 6BT, United Kingdom \\ ${ }^{5}$ Department of Electronic and Electrical Engineering, UCL, London, WC1E 7JE, United Kingdom \\ ${ }^{6}$ School of Mathematical and Physical Sciences, The University of Newcastle, Newcastle, Callaghan 2308, Australia
}

(Received 9 February 2016; revised manuscript received 12 April 2016; published 9 May 2016)

\begin{abstract}
We characterize the incorporation of $\mathrm{Ba}$ adatoms into the $\mathrm{Ge}(001)$ surface, resulting in the formation of one-dimensional structures with an internal $2 \times 3$ periodicity, after the deposition of Ba atoms at $970 \mathrm{~K}$ or at room temperature followed by a $770 \mathrm{~K}$ anneal. Scanning tunneling microscopy (STM) data were compared with theoretically simulated STM images generated by density functional theory electronic structure calculations. Excellent agreement between experiment and simulation was found when using an adopted structural model that assumes partial removal of the surface Ge dimers in the [1-10] surface direction and subsequent addition of a single $\mathrm{Ba}$ atom to the substrate second layer. Structural assignments for a number of defects observed within regions of the $2 \times 3$ reconstruction were also obtained.
\end{abstract}

DOI: 10.1103/PhysRevB.93.195304

\section{INTRODUCTION}

Future developments in microelectronic circuits will be underpinned by the ability to integrate different material platforms into existing Si-based technologies. One promising candidate for such integration is germanium $(\mathrm{Ge})$ [1]. This is because of the structural similarity of the $\mathrm{Ge}(001)$ surface to the technologically relevant $\mathrm{Si}(001)$ substrate $[2,3]$ and superior electronic properties of Ge compared to Si [1,4-6]. Progress in the field requires, however, and complete knowledge about technologically relevant processes on Ge surfaces such as controlled growth of low Ohmic contacts [7-11], formation of passivation layers [12-15], or structural and electronic functionalization [16,17].

The interaction of alkaline-earth metals such as strontium (Sr) or barium $(\mathrm{Ba})$ with the $\mathrm{Si}(001)$ and $\mathrm{Ge}(001)$ surfaces is fundamental to the controlled formation of high- $k$ surface layers [12-15,18]. At coverages below 0.5 monolayer (ML) these elements were shown to form two adsorption structures on $\mathrm{Si}(001)$ and $\mathrm{Ge}(001)$ —namely the on-top and incorporated phases $[15,19]$. The on-top phase on $\mathrm{Si}(001)$ was observed for the room temperature (RT) adsorption of $\mathrm{Ba}, \mathrm{Li}$, or $\mathrm{Sr}$ atoms in the form of randomly distributed chains of the adatoms that run across the Si dimer rows [19-23]. The analogous phase formed by the $\mathrm{Ba}$ adatoms on $\mathrm{Ge}(100)$ was demonstrated to consist of the Ba ad-dimers [24].

Deposition of the $\mathrm{Ba}$ or $\mathrm{Sr}$ atoms on $\mathrm{Si}(001)$ or $\mathrm{Ge}(001)$ substrates kept at elevated temperatures (usually above $600 \mathrm{~K}$ for $\mathrm{Ge}$ and $1000 \mathrm{~K}$ for $\mathrm{Si}$ ) was shown to result in the formation of the incorporated phase [22,25-29]. This phase is characterized by the adatom induced $2 \times 3$ surface reconstruction at coverages up to $1 / 6 \mathrm{ML}[28,30]$. It was demonstrated recently that annealing the on-top phase in the

\footnotetext{
*wojciech.koczorowski@put.poznan.pl
}

$\mathrm{Ba} / \mathrm{Ge}(001)$ adsorption system promotes the incorporation of the $\mathrm{Ba}$ adatoms into the substrate and transforms the on-top phase into the incorporated phase [15].

There has been a long debate regarding the structural models that could account for the $2 \times 3$ reconstruction of the incorporated phase observed on $\operatorname{Si}(001)[17,19,25-27,31]$. The formation of $2 \times 3$ is supported by the observations that the presence of the $\mathrm{Sr}$ adatoms on $\mathrm{Si}(001)$ reduces the activation energy barrier for the $\mathrm{Si}$ dimers to diffuse on the surface from 1.29 to $0.63 \mathrm{eV}$ (for 0.5 ML Sr coverage) [26]. It is anticipated, therefore, that at elevated temperatures there may be enough energy to relocate some of the $\mathrm{Si}(\mathrm{Ge})$ surface dimers on the substrate and incorporate the adatoms to form the $2 \times 3$ adsorption phase. This structural model initially proposed for the $\mathrm{Sr} / \mathrm{Si}(001)$ system has successfully been adopted for $\mathrm{Ba} / \mathrm{Si}(001)$ [31].

The main goal of the present paper is to analyze the formation processes of the incorporated phase in the $\mathrm{Ba} / \mathrm{Ge}(001)$ system and account for its structural properties by means of ultrahigh vacuum (UHV) scanning tunneling microscopy (STM) measurements and density functional theory (DFT) calculations. Two experimental procedures were used in the study and both are shown to lead to the formation of randomly distributed one-dimensional (1D) domains of the incorporated phases on $\mathrm{Ge}(001)$. In the first procedure the $\mathrm{Ge}(001)$ surface was held at $970 \mathrm{~K}$ (coverage $0.05 \mathrm{ML}$ ), while in the second, the $\mathrm{Ba}$ atoms were evaporated on the $\mathrm{Ge}(001)$ surface at RT (coverage $0.15 \mathrm{ML}$ ) followed by annealing at $770 \mathrm{~K}$ for $30 \mathrm{~min}$. It is shown that the $2 \times 3 \mathrm{Ba}$-induced reconstruction dominates the structure formed by adopting any of the two experimental procedures. The DFT calculations were performed adopting the $2 \times 3$ structural model proposed for the $\mathrm{Sr} / \mathrm{Si}(001)$ system $[26,32]$. We show that the simulated STM images properly account for the experimental STM data and especially allow for the identification of structural defects in the $2 \times 3$ Ba-induced periodicity typically observed in the 
experiment. This provides a strong argument that the adopted model properly describes the atomic structure of the $2 \times 3$ incorporated phase for the alkaline-earth metals on both the $\mathrm{Ge}(001)$ and $\mathrm{Si}(001)$ surfaces.

\section{METHODOLOGY}

\section{A. Experimental details}

Experiments were performed using a commercial Omicron Nanotechnology UHV system, equipped with UHV STM XA microscope with a variable temperature head. The base pressure in the vacuum system was kept below the $1 \times 10^{-10}$ mbar level (excluding Ge surface preparation).

$\mathrm{Ge}(001)$ samples $(3 \mathrm{~mm} \times 8 \mathrm{~mm})$ were cut out from a $\mathrm{Sb}$-doped, Ge wafer with resistivity of 1-10 $\Omega \mathrm{m}$. The samples were mounted on the standard Omicron direct heating sample holder. The reconstructed Ge(001) surface was obtained using several cycles of $\mathrm{Ar}^{+}$ion sputtering, followed by annealing at $970 \mathrm{~K}$ at UHV conditions [3]. The Ba atoms were evaporated on the surface by means of the Omicron EFM-3 e-beam evaporator, using a Mo crucible.

Two samples were analyzed. The first one was obtained by evaporating $\mathrm{Ba}$ atoms on the substrate whose temperature was maintained at $970 \mathrm{~K}$ for $10 \mathrm{~min}$ (including the deposition time of $104 \mathrm{~s}$ ) resulting in a coverage of $0.05 \mathrm{ML}$, and the second one by depositing $\mathrm{Ba}$ on the substrate kept at RT (Ba coverage of $0.15 \mathrm{ML}$ ) and annealed at $770 \mathrm{~K}$ for $30 \mathrm{~min}$. Coverages were estimated using proposed adsorption models for both samples [24]. Substrate temperature was controlled using an external pyrometer with an accuracy of $\pm 5 \mathrm{~K}$, focused at the middle part of the sample. Voltage bias-dependent STM images were taken at RT using electrochemically etched tungsten tips with the tunneling currents kept within the 20-150 pA range. The data processing of the topography STM images was performed using the WSXM software [33].

\section{B. Computational details}

Spin-polarized DFT calculations were performed using the VASP code $[34,35]$. The plane-waves basis set with the energy cutoff of $312.5 \mathrm{eV}$, projector augmented wave (PAW) potentials [36], the generalized gradient approximation (GGA) for correlation and exchange [37], and the PAW potentials were adopted in the calculations. The $2 \times 4$ and $4 \times 3$ surface unit cells were employed using asymmetric slabs (with the calculated bulk lattice constant of $5.78 \AA$ ) containing six layers and terminated on the bottom surface by hydrogen atoms. Four special $k$ points in the irreducible part of the surface Brillouin zone were used in the calculations. The energy convergence tolerance was $10^{-7} \mathrm{eV}$.

The surface charge and probability densities were calculated by integrating the local density of states function (ILDOS) over an energy range of \pm 1.0 and $\pm 2.0 \mathrm{eV}$ from the Fermi energy $\left(E_{F}\right)$. The obtained data are presented as $x$ $y z$ plots in which the height $z$ is associated with tracing out a selected ILDOS isosurface value and then converted into a gray scale. Such representations of the calculated densities can be interpreted as bias-dependent simulated STM images in the Tersoff-Hamann approximation [38].



FIG. 1. Ba-induced adsorption phases on Ge(001) (all images are $50 \times 50 \mathrm{~nm}^{2}$ ). Filled- (a) and empty- (b) state STM images of the incorporated phase formed after evaporating the $\mathrm{Ba}$ atoms at $970 \mathrm{~K}$ (arrows indicate the Ba-induced structures), (c) on-top phase formed after depositing Ba atoms at RT (marked with the arrows), and (d) incorporated phase obtained after annealing the on-top phase of (c) at $770 \mathrm{~K}$ for $30 \mathrm{~min}$ (arrows indicate the Ba-induced structures formed on the neighboring dimer rows).

\section{RESULTS AND DISCUSSIONS}

\section{A. Experimental STM data}

Figures 1(a) and 1(b) show filled- and empty-state STM images, respectively, of the incorporated phase obtained by evaporating $0.05 \mathrm{ML}$ of the $\mathrm{Ba}$ atoms on the $\mathrm{Ge}(001)$ substrate at $970 \mathrm{~K}$. Figure 1(c) shows an empty-state STM image of the on-top phase obtained by depositing $\sim 0.15 \mathrm{ML} B$ a on $\mathrm{Ge}(001)$ at RT. The empty-state STM image of Fig. 1(d) illustrates the effect of post evaporation annealing, in UHV, of the on-top phase of Fig. 1(c) at $770 \mathrm{~K}$ for $30 \mathrm{~min}$.

Close inspection of the empty-state data images presented in Figs. 1(b) and 1(d) shows that the incorporated phases formed via the two distinct experimental procedures are virtually identical. We observe that in both cases the randomly distributed 1D features (Ba lines) are formed along the $\mathrm{Ge}$ surface dimer rows and that the features are surrounded by atomically flat and clean portions of the $\mathrm{Ge}(001)$ substrate with a mixture of $p(2 \times 1)$ and $c(4 \times 2)$ surface reconstructions. The data presented in the empty-state STM images of Figs. 1(b) and 1(d) (and Fig. 2, see later) indicate that the oval-shaped bright protrusions in the incorporated line are regularly spaced and separated by $\sim 1.2$ (2) $\mathrm{nm}$ along the [1-10] direction, resulting in the local $2 \times 3$ periodicity. We also observe that the incorporated lines do not change their positions during scanning. This is in contrast to the on-top phase where the position of the individual $\mathrm{Ba}$ dimers in the chains can be changed during STM scanning [24]. This suggests that 


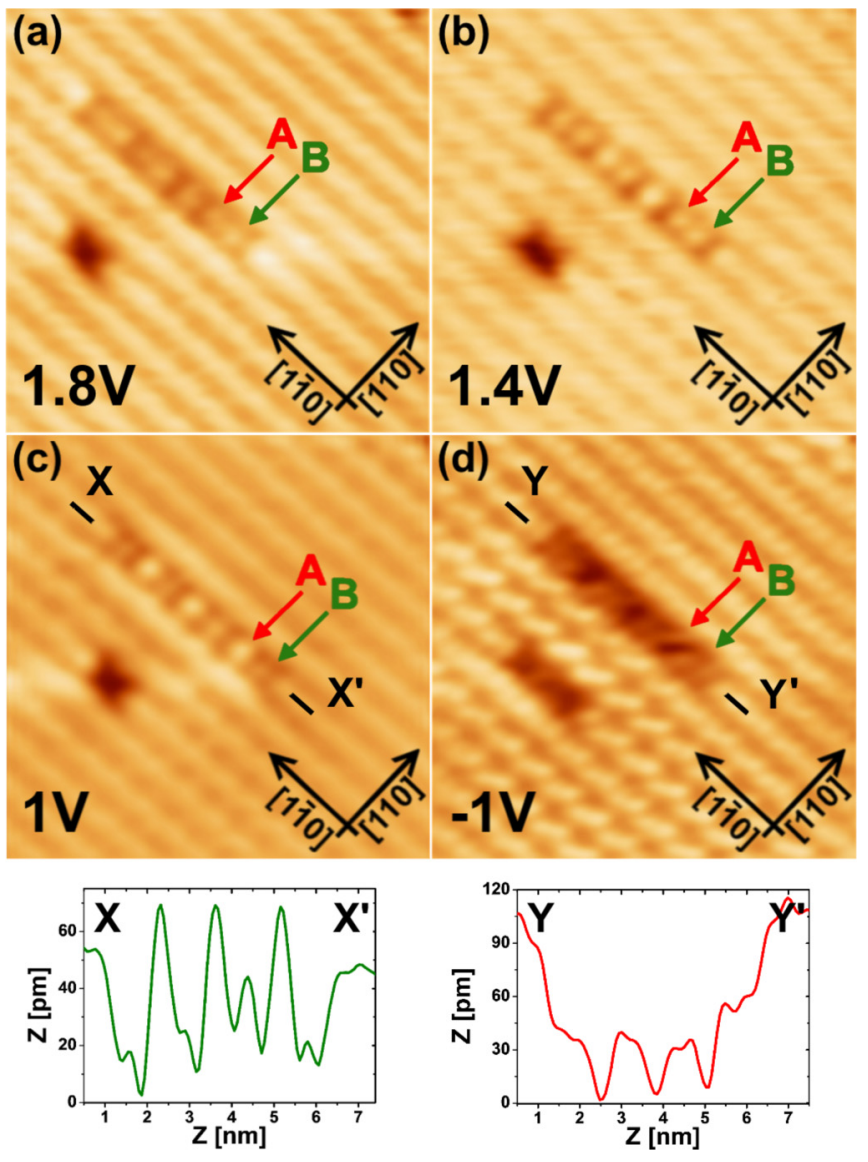

FIG. 2. Empty- (a-c) and filled- (d) state STM images $(10 \times$ $10 \mathrm{~nm}^{2}$ ) of an isolated Ba-incorporated line with $2 \times 3$ periodicity formed on $\mathrm{Ge}(001)$ after evaporation of $\mathrm{Ba}(0.05 \mathrm{ML}$ coverage) at $970 \mathrm{~K}$. The green and red arrows indicate features A and B, respectively, with their relative brightness changing with the bias voltage - compare $(\mathrm{a}, \mathrm{c})$ (for details see the text). Lover panel shows the cross section measured along the $X-X^{\prime}$ and $Y-Y^{\prime}$ lines shown in (c,d), respectively.

the binding energy of the $\mathrm{Ba}$ adatoms incorporated into the substrate is higher than that for the $\mathrm{Ba}$ adatoms in the on-top phase (at RT conditions). In addition, if the $2 \times 3 \mathrm{Ba}$ lines are incorporated next to each other on the neighboring surface dimer rows, the larger two-dimensional (2D) domains with the $2 \times 3$ periodicity are formed [see Fig. 1(d)] .

The bias-dependent STM images of an isolated Baincorporated line are shown in Fig. 2. The analysis of the empty-state images of Fig. 2 shows that the $2 \times 3$ unit cell consists of a pair of bright, oval-in-shape protrusions [marked with $\mathrm{A}$ and $\mathrm{B}$ in Figs. 2(a)-2(c)]. One can also observe that protrusion $\mathrm{A}$ is imaged darker than $\mathrm{B}$ at higher bias voltage [1.8 V, Fig. 2(a)], but becomes brighter than B for low bias voltage $[1 \mathrm{~V}$, Fig. 2(c)] and has similar brightness at medium bias voltages $[\sim 1.4 \mathrm{~V}$, Fig. 2 (b)]. By contrast, the filled-state STM images of the same structures do not exhibit any significant dependence on the bias voltage. The apparent height measurements in the filled-state STM image of Fig. 2(d) indicate that the incorporated lines are located $(50-80 \mathrm{pm})$ below the surrounding Ge surface dimers.

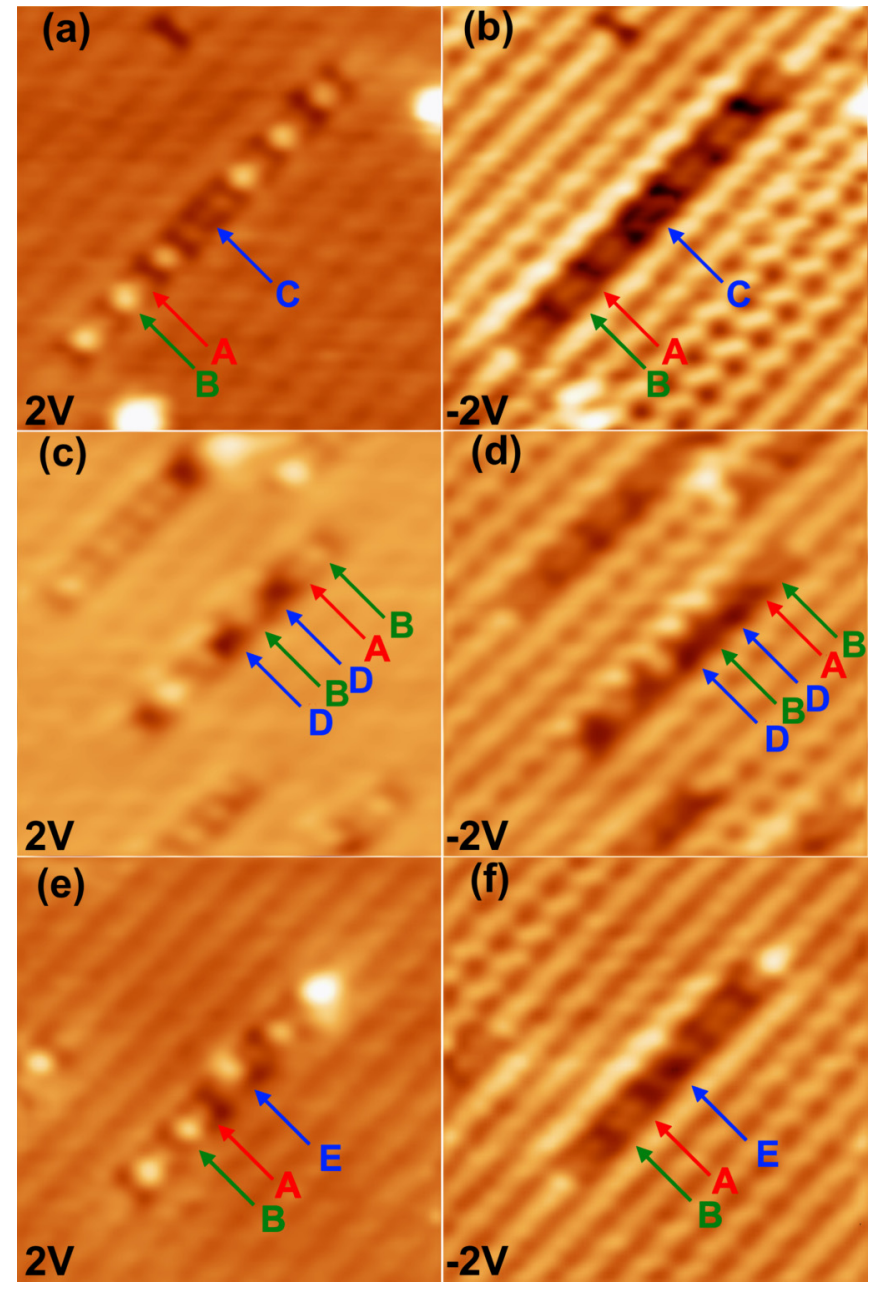

FIG. 3. Examples of the defects in the $2 \times 3$ incorporated line formed after evaporation of $0.05 \mathrm{ML}$ of $\mathrm{Ba}$ on the $\mathrm{Ge}(001)$ substrate at $970 \mathrm{~K}$. The features in the undefected lines within the $2 \times 3$ unit cell are marked as A and B. (a,b) show missing brighter feature B at $2 \mathrm{~V}$ bias (denoted by letter $\mathrm{C}$ ); (c,d) show structure with darker area in the incorporated line (denoted by letter D); (e,f) show brighter feature located at the valley bridge site between two Ge dimer rows (denoted by letter E). Size of all images is $10 \times 10 \mathrm{~nm}^{2}$.

The Ba-induced lines are frequently observed to be formed with defects, and those typically observed are shown in Fig. 3. The incorporated line with missing protrusion B is shown in the empty- and filled-state images in Figs. 3(a) and 3(b), respectively (marked by letter $\mathrm{C}$ ). We observe that this defect (defect $\mathrm{C}$ ) has no impact on the overall $2 \times 3$ periodicity along the line. Experimentally, the brightness of this protrusion is much less intensive than that marked with letter $B$ in the neighboring, defect-free $2 \times 3$ unit cells for biases 2 and $1.5 \mathrm{~V}$.

The second type of defect (defect D) is shown in the empty- and filled-state STM images of Figs. 3(c) and 3(d), respectively. The incorporated line visible at the center of these figures has two dark, extended features (marked by letter D) separated by the brighter feature (marked by letter B). In the filled-state STM image feature B becomes darker [see Fig. 3(d)]. In addition defect D is usually located at the end of 

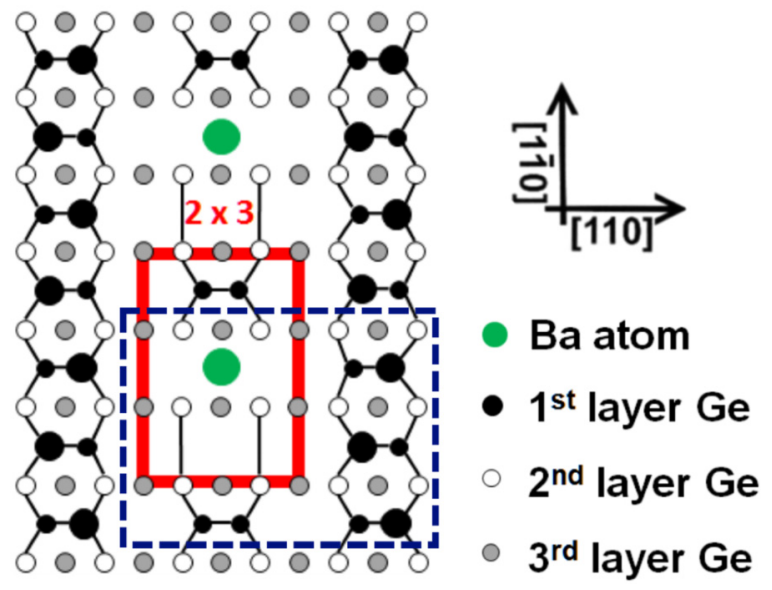

FIG. 4. A structural model of the $2 \times 3$ phase induced by the incorporation of the $\mathrm{Ba}$ adatom into the $\mathrm{Ge}(001) c(4 \times 2)$ substrate. The $2 \times 3$ unit cell is marked by the red (solid line) rectangle. The rectangle marked by the blue (dashed) line outlines the $4 \times 3$ surface unit cell used in the DFT calculations.

the incorporated line. We also note that defect C of Figs. 1(a) and $1(b)$ is observed less frequently than defect $\mathrm{D}$.

Finally, defect $E$ represented by an asymmetric protrusion in a single $2 \times 3$ unit cell is shown in Figs. 3(e) and 3(f) (marked by letter $\mathrm{E}$ ). The brightness of this protrusion is similar to the brightness of feature B in the empty-state STM images of the defect-free incorporated line (see Figs. 1 and 2); it is just shifted by about $0.4 \mathrm{~nm}$ in the [110] direction and is located closer to the neighboring, Ge surface dimer row. The filled-state image of this defect shows that its presence on the surface has no impact on the surrounding Ge surface structure; see Fig. 3(f).

\section{B. Modeling}

The complexity of the experimental STM images, especially their strong dependence on bias voltage and the presence of defects, makes it difficult to determine the atomic structures of the observed features. However, comparison of our experimental data with those reported for the $\mathrm{Sr} / \mathrm{Si}(001)$ system indicates that both sets of results are somewhat compatible $[26,27,32]$. It was shown for the $\mathrm{Sr} / \mathrm{Si}(001)$ system that $2 \times 3$ and $2 \times 6$ structures are much more stable than other models at low coverages [32]. As the energetics of the $\mathrm{Sr} / \mathrm{Si}(001)$ and $\mathrm{Ba} / \mathrm{Si}(001)$ systems are very similar [31] we will adopt for the $\mathrm{Ba} / \mathrm{Ge}(001)$ system the structural model proposed for the $\mathrm{Sr} / \mathrm{Si}(001)$ system [26,32]. We will further verify the validity of the adopted model and associate the atomic configurations to the defects identified in the Bainduced surface reconstructions.

The adopted structural model is shown in Fig. 4. It assumes that the Ba-induced $2 \times 3$ surface unit cell with initially three adjacent Ge dimers along the dimer row is formed by removing two of them and exposing four second-layer Ge atoms. These atoms form two new, second-layer Ge dimers. The Ba adatom is placed into this "hollow" site in the vicinity of the remaining first-layer Ge dimer and the two, newly formed second-layer Ge dimers.

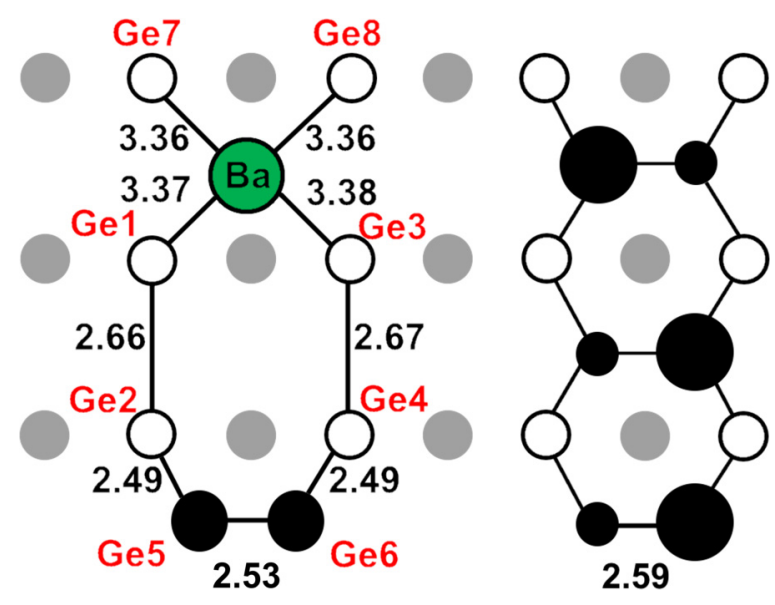

FIG. 5. Top view of the considered structural model of the Ba $2 \times 3$ incorporated phase on $\mathrm{Ge}(001)$. The calculated bond lengths (in $\AA$ ) are also shown. First-layer Ge: black circles; second-layer Ge: white circles; third-layer Ge: gray circles; Ba: green circle.

Figure 5 shows the details of the optimized geometry. The data demonstrate that the $\mathrm{Ba}$ adatom incorporated into the surface is bonded to four surrounding second-layer Ge atoms (Ge1, Ge3, Ge7, and Ge8), with the bond lengths of 3.36$3.38 \AA$. The Ge surface dimer within the $2 \times 3$ unit cell has its buckling angle reduced from $19.4^{\circ}$ (bare, ideal surface) to $0.2^{\circ}$ and its length is reduced from $2.59 \AA$ for the bare $\mathrm{Ge}$ dimers to $2.53 \AA$. The two second-layer Ge dimers (Ge1$\mathrm{Ge} 2, \mathrm{Ge} 3-\mathrm{Ge} 4)$ are also flat with the bond lengths of $2.67 \AA$. The DFT calculations were carried out for the $4 \times 3$ surface unit cell shown in Fig. 4. As a result on the unchemisorbed dimer row two Ge dimers are buckled in the same direction giving the local $p(2 \times 1)$ surface reconstruction (see Fig. 6). However, this does not affect the geometry of the $2 \times 3$ unit
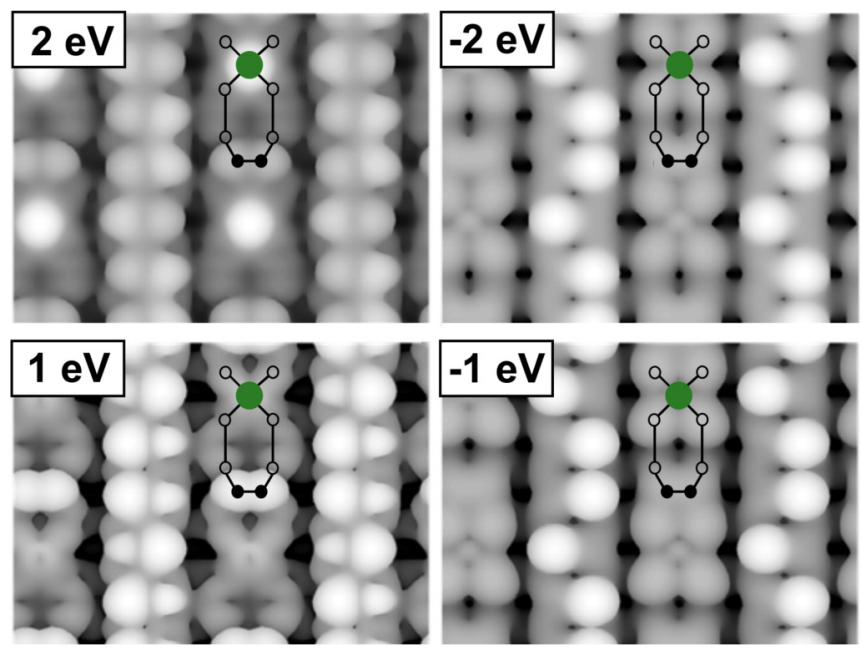

FIG. 6. Simulated empty- (left) and filled- (right) state STM images of the Ba-incorporated phase on $\mathrm{Ge}(001)$ [four identical $(4 \times 3)$ surface unit cells are reproduced in each image]. On the unchemisorbed dimer row two dimers are buckled in the same direction giving the local $p(2 \times 1)$ surface reconstruction. This is because a $4 \times 3$ surface unit cell was used in the DFT calculations (see Fig. 4). The same applies to Figs. 7-9. 


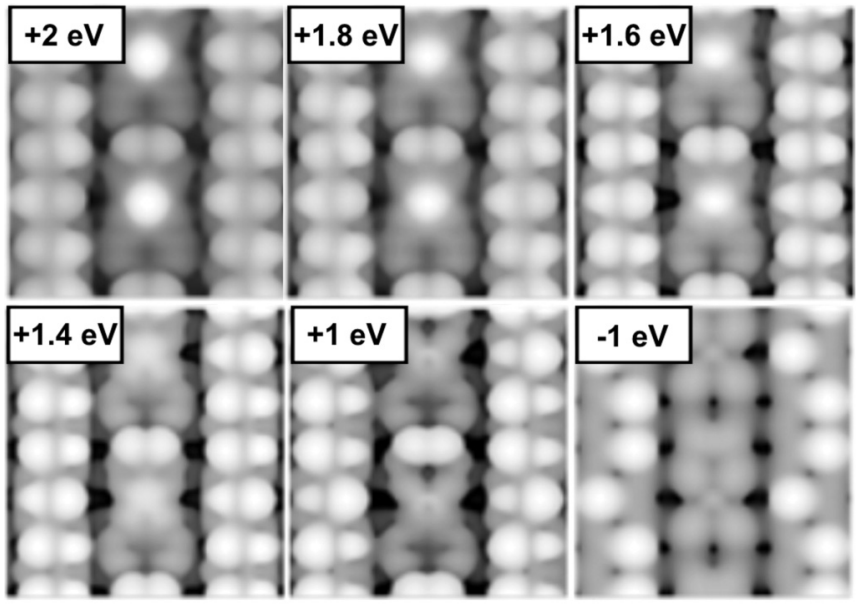

FIG. 7. Bias dependence in the simulated STM empty- $(+e V)$ and filled- $(-\mathrm{eV})$ state images of the $2 \times 3 \mathrm{Ba}$-incorporated phase on $\mathrm{Ge}(001)$.

cell induced by the adsorption of Ba (Fig. 4), as the detailed atomic geometry of the rows neighboring the chemisorbed ones has little or no effect on the energetics and stability of the adsorption-induced geometries.

The calculated electronic structure indicates that the $\mathrm{Ba}$ adatom donates its two $6 \mathrm{~s}$ electrons to the two dangling bonds of the second-layer Ge dimers. These dimers are flattened with quite large bond length $(2.67 \AA)$ because they also accept two extra electrons from the occupied dangling bond of the first-layer Ge dimer. This results in this first-layer dimer being also flat with the shorter bond length of $2.53 \AA$. The effect of such charge transformation is clearly seen in the simulated STM images presented in Fig. 6.

At positive bias (integration range of $+1 \mathrm{eV}$ above the Fermi level $E_{F}$; Fig. 6, bottom left) the Ba adatoms are not visible and the bright, oval protrusion is associated with the first-layer Ge dimer (Ge5-Ge6 in Fig. 5). At higher positive bias (integration range of $+2 \mathrm{eV}$ above $E_{F}$; Fig. 6, top left) the bright feature located on the $\mathrm{Ba}$ adatom dominates the image. The progressive changes in relative intensities of both protrusions as a function of the integration range (bias voltage) in the simulated empty-state STM images are shown in Fig. 7. The observed bias dependence is consistent with the experimental data (see Fig. 2) and allows us to identify protrusion $\mathrm{A}$ with the first-layer Ge dimer and protrusion B with the $\mathrm{Ba}$ adatom. Similar effects in the bias-dependent STM imaging have been reported by Lukanov et al. [39] for the $\mathrm{Sr} / \mathrm{Ge}(001)$ adsorption system. The authors showed that in the empty-state STM images the brightness of the $\mathrm{Sr}$ atoms incorporated into the $\mathrm{Ge}(001)$ substrate increases with increasing bias voltage [39].

The simulated filled-state STM images shown in Fig. 6 (right) are virtually independent of the LDOS integration range (bias voltage). This is also consistent with experiment. We observe that in these images the site where the $\mathrm{Ba}$ adatom is adsorbed and the first-layer Ge dimer are imaged dark. This is consistent with the electronic structure calculations that indicate the electron transfer from the Ba adatom and the (a)

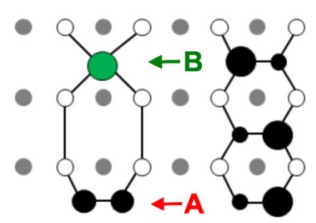

(b)

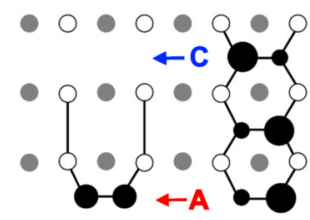

(c)

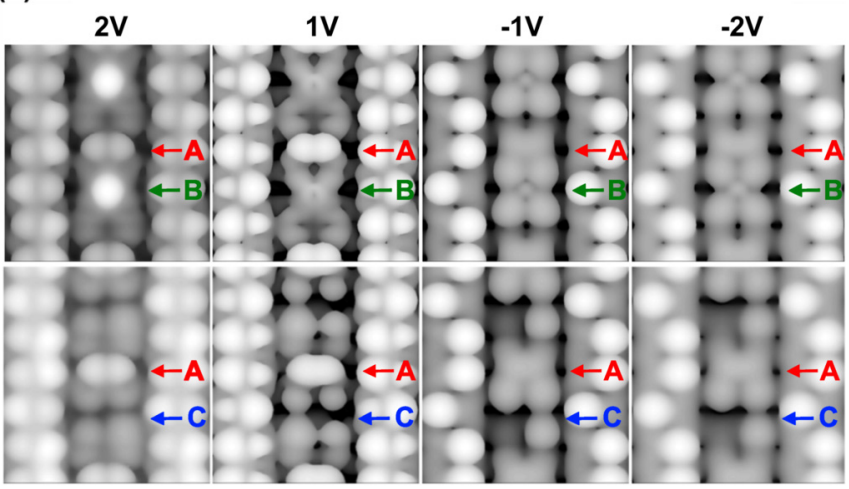

(d)

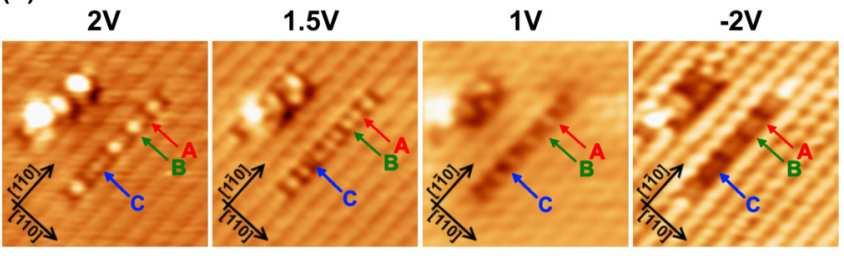

FIG. 8. Structural models for the undisturbed and defected $2 \times 3$ incorporated phase on $\mathrm{Ge}(001)$ : (a) undisturbed $2 \times 3$ phase; (b) the $\mathrm{C}$ defect of Fig. 3(a). (c) DFT simulations of empty- and filled-state images of undefected $2 \times 3$ phase (upper panel), and phase without incorporated $\mathrm{Ba}$ adatom (lower panel). (d) Experimental STM images taken at different bias voltages. Positions of the Ge dimer and $\mathrm{Ba}$ atom in the undisturbed $2 \times 3$ unit cell marked with $\mathrm{A}$ and $\mathrm{B}$, respectively. Defect position is marked with the blue arrow and letter $\mathrm{C}$.

$p_{z}$-like states of the first-layer Ge dimer to the second-layer Ge dimers.

Understanding the structural details of the undisturbed $2 \times 3$ phase allows us to model the atomic structures of the defects shown in Fig. 3. The $\mathrm{C}$ defect of Figs. 3(a) and 3 (b) has been identified as a missing $\mathrm{Ba}$ adatom. The atomic arrangement for the undisturbed $2 \times 3$ structure and the defect are presented in Figs. 8(a) and 8(b), respectively. The corresponding simulated STM images of this defect are shown in Fig. 8(c). One can observe that the visible feature within the defected unit cell originates from the first-layer $\mathrm{Ge}$ dimer located in the incorporated structure and its brightness increases with decreasing bias voltage in the empty-state images. This is consistent with the experimental data; see Fig. 8(d).

The D defect of Figs. 3(c) and 3(d) has been identified as a missing first-layer Ge dimer originally located in the incorporated line. The atomic arrangement for the ideal $2 \times 3$ Ba-induced structure and this defect are shown in Figs. 9(a) and 9(b), respectively. The simulated STM images of the $\mathrm{D}$ defect and the undisturbed $2 \times 3$ structure are shown in Fig. 9(c). We observe that in the simulated empty-state STM 
(a)

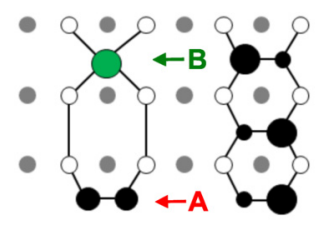

(c)

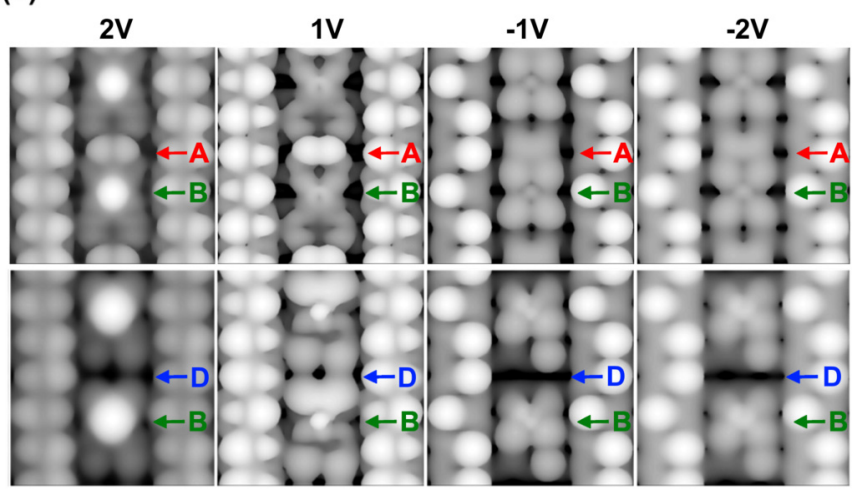

(d)

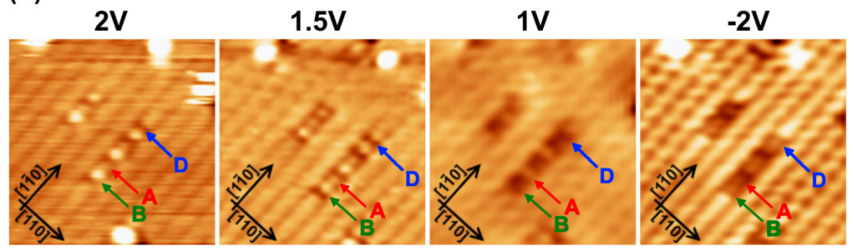

FIG. 9. Structural models for the undisturbed and defected $2 \times 3$ incorporated phase on $\mathrm{Ge}(001)$ : (a) undisturbed $2 \times 3$ phase; (b) the D defect of Fig. 3(b). (c) DFT simulations of empty- and filled-state images of undefected $2 \times 3$ phase (upper panel), and phase without incorporated $\mathrm{Ba}$ adatom (lower panel). (d) experimental STM images taken at different bias voltages. Positions of the $\mathrm{Ge}$ dimer and $\mathrm{Ba}$ atom in the undisturbed $2 \times 3$ unit cell marked with A and B, respectively. Defect position is marked with the blue arrow and letter D.

images (lower panel in Fig. 9) the feature attributed to the Ba atom becomes brighter with increasing bias voltage. This is also consistent with the experimental STM images in Fig. 9(d). Finally, based on the above assignments we conclude that the E defect of Figs. 3(e) and 3(f) can be attributed to the Ba atom adsorbed at the valley bridge site between the surface Ge dimer rows, one of which has the Ba-incorporated line.

\section{SUMMARY}

We studied the atomic structure of the $2 \times 3$ incorporated phase of $\mathrm{Ba}$ on $\mathrm{Ge}(001)$ at low coverage using STM and DFT. Surfaces exhibiting the incorporated phase of Ba were experimentally obtained by two different methods and were shown to have the same structural properties. We found excellent agreement between our experimental STM data and the simulated STM images obtained based on the structural model initially proposed for the $\mathrm{Sr} / \mathrm{Si}(001)$ system. We therefore conclude that the periodic $2 \times 3 \mathrm{Ba}$-incorporated phase on $\mathrm{Ge}(001)$ is structurally identical to that on $\mathrm{Sr} / \mathrm{Si}(100)[26,27,32]$ and as such is not consistent with the early models proposed by $\mathrm{Du}$ et al. [17], Hu et al. [19], and Ojima et al. [25] Furthermore, comparison of our STM and DFT data enabled us to assign structure of a number of defects observed within regions of Ba-induced $2 \times 3$ reconstruction.

\section{ACKNOWLEDGMENTS}

W.K. and N.J.C. acknowledge EPSRC Grant No. EP/I02865X/1. W.K., M.W.R., L.J., and R.C. acknowledge the Polish National Science Centre (Project No. N-N-202195840) and Polish Ministry of Science and Higher Education (Project No. 06/62/DSPB/0216) for support. Innovations for High Performance Microelectronics (IHP GmbH) institute in Frankfurt (Oder), Germany is acknowledged for support in experimental studies. Numerical calculations reported in this work have been performed at the Interdisciplinary Centre of Mathematical and Computational Modelling of the University of Warsaw within Grant No. G44-10.

W.K. and A.P. contributed equally to this work.
[1] C. Claeys and E. Simoen, Germanium-Based Technologies: From Materials to Devices (Elsevier, Leuven, Belgium, 2007).

[2] R. J. Hamers and U. K. Kohler, J. Vac. Sci. Technol. A 7, 2854 (1989).

[3] H. J. W. Zandvliet, Phys. Rep. 388, 1 (2003).

[4] M. W. Radny, G. A. Shah, S. R. Schofield, P. V. Smith, and N. J. Curson, Phys. Rev. Lett. 100, 246807 (2008).

[5] P. V. Smith, M. Hermanowicz, G. A. Shah, and M. W. Radny, Comput. Mater. Sci. 54, 37 (2012).

[6] P. V. Smith, M. W. Radny, and G. A. Shah, J. Phys.: Condens. Matter 25, 056007 (2013).

[7] A. Chawanda, C. Nyamhere, F. D. Auret, W. Mtangi, T. T. Hlatshwayo, M. Diale, and J. M. Nel, Physica B (Amsterdam, Neth.) 404, 4482 (2009).

[8] A. Chawanda, C. Nyamhere, F. D. Auret, W. Mtangi, M. Diale, and J. M. Nel, Phys. Status Solidi C 7, 248 (2010).
[9] R. E. Camacho-Aguilera, Y. Cai, N. Patel, J. T. Bessette, M. Romagnoli, L. C. Kimerling, and J. Michel, Opt. Express 20, 11316 (2012).

[10] T. Grzela, W. Koczorowski, G. Capellini, R. Czajka, M. W. Radny, N. J. Curson, S. R. Schofield, M. A. Schubert, and T. Schroeder, J. Appl. Phys. 115, 074307 (2014).

[11] T. Grzela, G. Capellini, W. Koczorowski, M. A. Schubert, R. Czajka, N. J. Curson, I. Heidmann, T. Schmidt, J. Falta, and T. Schroeder, Nanotechnology 26, 385701 (2015).

[12] R. A. McKee, F. J. Walker, and M. F. Chisholm, Science 293, 468 (2001).

[13] J. W. Reiner, A. M. Kolpak, Y. Segal, K. F. Garrity, S. IsmailBeigi, Ch. H. Ahn, and F. J. Walker, Adv. Mater. 22, 2919 (2010).

[14] C. Merckling, G. Saint-Girons, C. Botella, G. Hollinger, M. Heyns, J. Dekoster, and M. Caymax, Appl. Phys. Lett. 98, 092901 (2011). 
[15] W. Koczorowski, T. Grzela, M. W. Radny, S. R. Schofield, G. Capellini, R. Czajka, T. Schroeder, and N. J. Curson, Nanotechnology 26, 155701 (2015).

[16] G. Scappucci, G. Capellini, W. C. T. Lee, and M. Y. Simmons, Nanotechnology 20, 495302 (2009).

[17] W. Du, B. Wang, L. Xu, Z. Hu, X. Cui, B. C. Pan, J. Yang, and J. G. Hou, J. Chem. Phys. 129, 164707 (2008).

[18] R. A. McKee, F. J. Walker, and M. F. Chisholm, Phys. Rev. Lett. 81, 3014 (1998).

[19] X. Hu, X. Yao, C. Peterson, D. Sarid, and Z. Yu, Surf. Sci. 445, 256 (2000)

[20] Y. Hasegawa, I. Kamiya, T. Hashizume, T. Sakurai, H. Tochihara, M. Kubota, and Y. Murata, Phys. Rev. B 41, 9688 (1990).

[21] R. Bakhtizin, J. Vac. Sci. Technol. B 14, 1000 (1996).

[22] R. Bakhtizin and J. Kishimoto, Appl. Surf. Sci. 94-95, 478 (1996).

[23] X. Hu, X. Yao, C. A. Peterson, D. Sarid, Z. Yu, J. Wang, D. S. Marshall, J. A. Curless, J. Ramdani, R. Droopad, J. A. Hallmark, and W. J. Ooms, Surf. Sci. 457, L391 (2000).

[24] W. Koczorowski, A. Puchalska, T. Grzela, M. W. Radny, L. Jurczyszyn, S. R. Schofield, R. Czajka, and N. J. Curson, Phys. Rev. B 91, 235319 (2015).

[25] K. Ojima, M. Yoshimura, and K. Ueda, Surf. Sci. 491, 169 (2001).

[26] J. W. Reiner, K. F. Garrity, F. J. Walker, S. Ismail-Beigi, and C. H. Ahn, Phys. Rev. Lett. 101, 105503 (2008).
[27] J. He, G. Zhang, J. Guo, Q. Guo, and K. Wu, J. Appl. Phys. 109, $083522(2011)$

[28] B. R. Lukanov, J. W. Reiner, F. J. Walker, C. H. Ahn, and E. I. Altman, Phys. Rev. B 84, 075330 (2011).

[29] B. R. Lukanov, K. F. Garrity, S. Ismail-Beigi, and E. I. Altman, Phys. Rev. B 85, 195316 (2012).

[30] J. W. Reiner, Y. Segal, K. F. Garrity, H. Hong, S. Ismail-Beigi, C. H. Ahn, and F. J. Walker, J. Vac. Sci. Technol. B 27, 2015 (2009).

[31] M. Kuzmin, M. P. J. Punkkinen, P. Laukkanen, J. J. K. Lång, J. Dahl, L. Vitos, and K. Kokko, J. Phys. Chem. C 118, 1894 (2014).

[32] K. F. Garrity and S. Ismail-Beigi, Phys. Rev. B 80, 085306 (2009).

[33] I. Horcas, R. Fernández, J. M. Gómez-Rodríguez, J. Colchero, J. Gómez-Herrero, and A. M. Baro, Rev. Sci. Instrum. 78, 013705 (2007).

[34] G. Kresse and J. Furthmuller, Comput. Mater. Sci. 6, 15 (1996).

[35] G. Kresse and J. Furthmuller, Phys. Rev. B 54, 11169 (1996).

[36] P. E. Blöchl, Phys. Rev. B 50, 17953 (1994).

[37] J. P. Perdew, J. A. Chevary, S. H. Vosko, K. A. Jackson, M. R. Pederson, D. J. Singh, and C. Fiolhais, Phys. Rev. B 46, 6671 (1992).

[38] J. Tersoff and D. R. Hamann, Phys. Rev. Lett. 50, 1998 (1983).

[39] B. R. Lukanov, K. F. Garrity, S. Ismail-Beigi, and E. I. Altman, Phys. Rev. B 89, 155319 (2014). 